\title{
Increasing Utilization of Redundant Virtual Array for DOA Estimation Based on Coprime Array
}

\author{
Chenghong Zhan $\mathbb{D D}^{1,2}$ Guoping $\mathrm{Hu} \mathbb{D}^{1},{ }^{1}$ Zixin Zhang $\mathbb{D}^{1,2}$ and Ziang Feng ${ }^{1}$ \\ ${ }^{1}$ Air and Missile Defense College, Air Force Engineering University, Xi'an 710051, China \\ ${ }^{2}$ Graduate College, Air Force Engineering University, Xi'an 710051, China \\ Correspondence should be addressed to Guoping Hu; mma18207171897@163.com
}

Received 13 September 2020; Revised 22 October 2020; Accepted 29 October 2020; Published 30 November 2020

Academic Editor: Alessandro Gasparetto

Copyright (c) 2020 Chenghong Zhan et al. This is an open access article distributed under the Creative Commons Attribution License, which permits unrestricted use, distribution, and reproduction in any medium, provided the original work is properly cited.

\begin{abstract}
In this paper, we initiated a method to estimate the direction of arrival (DOA) of far-field, narrowband, and incoherent targets using coprime array. First, we proposed a coprime array structure and analysed the distribution of difference coarray (DCA). The degrees of freedom (DOF) of the proposed coprime array became clearer by referring to the DCA conception. However, previous algorithm only uses the continuous virtual array, which causes the virtual array elements in the repeated position being abandoned. Therefore, the paper analyses the distribution of virtual array based on DCA conception and averages the receiving signal on these redundant virtual array elements to increase the utilization of receiving data. As a result, the algorithm has high precision in parameter estimation. Simulation results have shown the superiority of the proposed algorithm.
\end{abstract}

\section{Introduction}

Because sparse arrays can overcome the limitation of interelement spacing, direction of arrival (DOA) estimation based on it has attracted great attention. Compared to the uniform linear arrays (ULAs), sparse arrays, such as minimum redundancy array (MRA) [1], nested array (NA) [2, 3], and coprime array (CPA) [4], have great advantages in improving estimation accuracy, enhancing angle resolution, and reducing physical cost [5-8]. Larger array element spacing expands the virtual aperture, which leads to the rise in the number of detectable sources and angle resolution. In addition, it can also effectively suppress the mutual coupling effect of array elements and the coherence of received noise. Thus, improving the estimation, low redundancy can effectively reduce wastage of resources and accelerate operation [9-11].

Coprime arrays have more DOFs than ULAs and have no mutual coupling problems compared with nested arrays [12]. Furthermore, it has closed form expression of freedom extension degree and systematic array structure design. Therefore, DOA estimation based on coprime arrays has become a research hotspot. Most algorithms with coprime arrays derive extended virtual array and operate the corresponding signals to estimate DOA [13-16]. In [17], the paper proposes a modified Toeplitz matrix reconstruction method using a random nonzero row of the covariance matrix in the virtual uniform linear array. Besides, a full rank covariance matrix is obtained by restructuring a Toeplitz matrix. In [18], the author recovered the Toeplitz matrix by solving a nuclear norm minimization problem and decohered the signal. In addition, the discrete virtual array elements are utilized by interpolating virtual elements. However, the virtual arrays in repeated position were abandoned. In [16], a generalized coprime array configuration for DOA estimation is proposed. Based on generalized sum and difference coarray (GSDC) concept, the proposed configuration enlarges the interelement space, and hence, the DOFs of the arrays are increased. Besides, there are a large number of virtual arrays in the same position which are not utilized. On the other hand, in [19], an algorithm with low computational complexity and high estimation accuracy was initiated. The method first used estimates signal parameters via rotational invariance 
technique (ESPRIT) algorithm. Then, the search scope was narrowed down based on the initial results. Finally, more accurate estimation results were obtained by multiple signal classification (MUSIC). Similarly, in order to reduce the computational cost, the authors of [20] propose a successful signal subspace fitting (SSF) method from a computationally efficient perspective. All of the algorithms above focused either on improving the array structure to obtain higher DOFs and larger virtual array aperture or on reducing the complexity by avoiding spatial spectrum search and so on. Obviously, the redundant virtual elements in the same position were not utilized, and their data are wasted.

In this paper, we take the coprime array with the difference coarray (DCA) as an example to illustrate the redundant virtual array elements distribution and then average the virtual elements in the repeated position to increase the utilization efficiency of received signal data. Consequently, the snapshots of received signal have increased, and the DOA estimation performance of targets is improved noticeably.

\section{Coprime Array Signal Model}

As shown in Figure 1, a coprime array structure consists of two uniform linear subarrays. Subarray 1 has $N$ sensors with an interelement spacing $N$. The other subarray has $2 M-1$ sensors with interelement spacing $M$. Mand $N$ are a pair of coprime integers $(M<N)$. We assume the unit of interelement spacing is $d$, where $d=\lambda / 2$ is half-wavelength.

According to Figure 1, sensors' location is expressed as follows:

$$
S=\{\text { Mnd, } 0 \leq n \leq N-1\} \cup\{\mathrm{Nmd}, 0 \leq m \leq 2 M-1\} .
$$

We assume narrowband and uncorrelated sources in the far-field from distinct angles impinging on the array with sensors. The output of the received signal after the matched filter can be denoted as

$$
\mathbf{X}(t)=\mathbf{A S}(t)+\mathbf{N}(t)
$$

In the above equation, $\mathbf{S}(t)=\left[s_{1}(t), s_{2}(t), \ldots, s_{K}(t)\right]^{T}$, where $s_{k}(t)$ is the reflection coefficient of the $k$ th target. $\mathrm{N}(\mathrm{t})$ is the complex-valued additive white Gaussian noise matrix with mean value 0 and variance $\sigma^{2}$. Ais the steering vector which can be denoted as

$$
\mathbf{A}=\left[\mathbf{a}\left(\theta_{1}\right), \mathbf{a}\left(\theta_{2}\right), \ldots, \mathbf{a}\left(\theta_{K}\right)\right],
$$

where $\mathbf{a}\left(\theta_{k}\right)$ can be expressed as

$$
\mathbf{a}\left(\theta_{k}\right)=\left[1, e^{-j \pi \sin \left(\theta_{k}\right)}, e^{-j \pi M \sin \left(\theta_{k}\right)}, \ldots, e^{-j \pi(N-1) M \sin \left(\theta_{k}\right)}, e^{-j \pi N \sin \left(\theta_{k}\right)}, \ldots, e^{-j \pi(2 M-1) N \sin \left(\theta_{k}\right)}\right]^{T}
$$

Then, the covariance matrix can be given as

$$
\mathbf{R}=\mathbf{E}\left[\mathbf{X}(t) \mathbf{X}^{H}(t)\right]=\mathbf{A R}_{S} \mathbf{A}^{H}+\sigma^{2} \mathbf{I}_{N \times N},
$$

where $\mathbf{R}_{S}=E\left[\mathbf{S}(t) \mathbf{S}^{H}(t)\right]$ represents the target covariance matrix. $\mathbf{E}[\cdot]$ denotes mathematical expectation; $[\cdot]^{H}$ denotes conjugate transpose; $\sigma^{2}$ denotes the energy of the noise; and $\mathbf{I}_{N \times N}$ is $N \times N$ dimensional identity matrix. Because the signal is incoherent, $\mathbf{R}_{S}=E\left[\mathbf{S}(t) \mathbf{S}^{H}(t)\right]$ can be given as

$$
\mathbf{R}_{S}=\mathbf{E}\left[\mathbf{S}(t) \mathbf{S}^{H}(t)\right]=\operatorname{diag}\left[\sigma_{1}^{2}, \sigma_{2}^{2}, \ldots, \sigma_{K}^{2}\right],
$$

where the $\sigma_{k}^{2}$ denotes the power of the signal.

In practice, the signal covariance matrix is often estimated with $L$ snapshots.

$$
\widehat{\mathbf{R}}=\frac{1}{L} \sum_{t=1}^{L} \mathbf{X}(t) \mathbf{X}^{H}(t)
$$

$\widehat{\mathbf{R}}$ needs to be vectorized to obtain the virtual array elements. Consequently, the vectorizing covariance matrix of signal can be expressed as

$$
\begin{aligned}
\mathbf{r} & =\operatorname{vec}(\widehat{\mathbf{R}})=\left(\mathbf{A}^{*} \circ \mathbf{A}\right) \mathbf{p}+\sigma^{2} \operatorname{vec}\left(\mathbf{I}_{N \times N}\right) \\
& =\mathbf{B} \mathbf{p}+\sigma^{2} \operatorname{vec}\left(\mathbf{I}_{N \times N}\right),
\end{aligned}
$$

where $\operatorname{vec}(\cdot)$ denotes vectorizing the covariance matrix, $(\cdot)^{*}$ denotes conjugate operation, and $\circ$ denotes Khatri-Rao product. The virtual array elements are formed from steering vector $\mathbf{B}=\mathbf{A}^{*} \circ \mathbf{A}$. Then, combining formulae (3) and (8), the DAC conception can be expressed as

$$
\begin{aligned}
B & =\mathbf{A}^{*} \circ \mathbf{A} \\
& =\left[\mathbf{a}^{*}\left(\theta_{1}\right) \otimes \mathbf{a}\left(\theta_{1}\right), \mathbf{a}^{*}\left(\theta_{2}\right) \otimes \mathbf{a}\left(\theta_{2}\right), \ldots, \mathbf{a}^{*}\left(\theta_{K}\right) \otimes \mathbf{a}\left(\theta_{K}\right)\right],
\end{aligned}
$$

where $\otimes$ denotes the Kronecker product. Thus, vectorizing the covariance matrix $\widehat{\mathbf{R}}$ is an effective method for obtaining the virtual array and extending the array's aperture.

We express the virtual array elements as $S_{\mathrm{CPA}}$, and then it can be represented as

$$
\begin{aligned}
S_{\mathrm{CPA}} & =\left\{d_{n}-d_{\tilde{n}}, d_{n}, d_{\tilde{n}} \in P_{\mathrm{CPA}}\right\}=S_{s} \cup S_{c}, \\
S_{s} & =\{ \pm M n, \quad n=0, \ldots, N-1\} \cup\{ \pm N m, \quad m=0, \ldots, 2 M-1\}, \\
S_{c} & =\{ \pm(M n-N m), \quad m=0,1, \ldots, 2 M-1, n=0,1, \ldots, N-1\},
\end{aligned}
$$




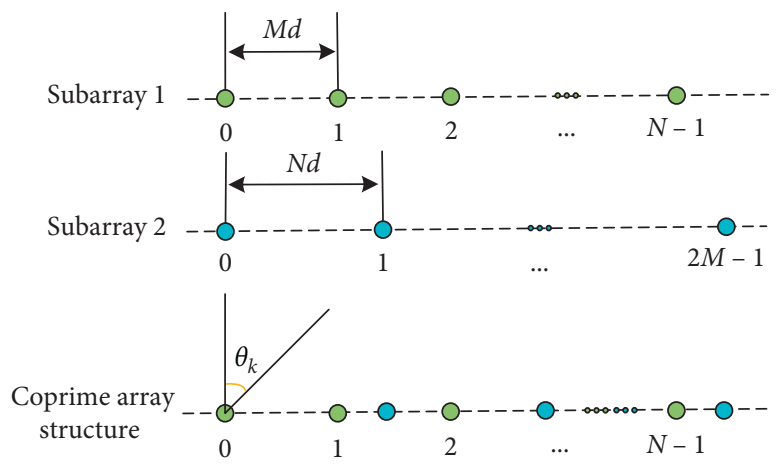

Figure 1: Coprime array MIMO radar structure.

where $S_{s}$ denotes the self-difference set and $S_{c}$ denotes the cross-difference set.
From $S_{s} \subset S_{c}$, formula (10) can be simplified as

$$
S_{\mathrm{CPA}}=\{ \pm(M n-N m), \quad m=0,1, \ldots, 2 M-1, n=0,1, \ldots, N-1\}
$$

From formula (11), DOFs of the virtual array is $O(M N)$ that only needs $2 M+N-1$ physical sensors. It is obvious that the virtual array elements are discontinuous. For example, when $M=2, N=3$, the continuous range of virtual elements is $[-7,7]$. Next, the distribution and application of virtual redundant elements are discussed.

\section{Improved Algorithm for Coprime Array}

In this section, we will compare traditional algorithms based on difference coarray and reuse the redundant virtual elements.

3.1. Review of Traditional Algorithm. In equations (8) and (9), the virtual elements formed by the vectorization of the signal covariance matrix are reordered, and redundant virtual elements are deleted. Then, a new steering vector of the single snapshot can be acquired and is marked as signal $\widehat{B}$, which is expressed as

$$
\widehat{B}=\left[\begin{array}{cccc}
e^{-j \pi N_{\text {Max }} \sin \theta_{1}} & e^{-j \pi N_{\text {Max }} \sin \theta_{2}} & \cdots & e^{-j \pi N_{M a x} \sin \theta_{K}} \\
\vdots & \vdots & \ddots & \vdots \\
e^{-j \pi \sin \theta_{1}} & e^{-j \pi \sin \theta_{2}} & \cdots & e^{-j \pi \sin \theta_{K}} \\
1 & 1 & \cdots & 1 \\
e^{j \pi \sin \theta_{1}} & e^{j \pi \sin \theta_{2}} & \ddots & e^{j \pi \sin \theta_{K}} \\
\vdots & \vdots & \vdots & \vdots \\
e^{j \pi N_{\text {Max }} \sin \theta_{1}} & e^{j \pi N_{\text {Max }} \sin \theta_{2}} & \cdots & e^{j \pi N_{\text {Max }} \sin \theta_{K}}
\end{array}\right],
$$

where $N_{\text {Max }}=M N+M-1$ denotes the maximum position of extended continuous virtual elements. Correspondingly, the range of the virtual array elements is $\left[-N_{\mathrm{Max}}, N_{\mathrm{Max}}\right]$.

Figure 2 shows the distribution of virtual array elements when $M=2$ and $N=3$.
In Figure 2, there are many redundant virtual array elements in the repeated position.

In the previous algorithm, redundant virtual elements in duplicate positions are deleted and reordered. In addition, the Toeplitz matrix method is used to eliminate the single snapshot effect and obtain a new signal covariance matrix.

The new single snapshot signal is marked as $\mathbf{r}_{0}$. Based on formulae (8) and (12), $\mathbf{r}_{0}$ can be expressed as

$$
\mathbf{r}_{0}=\widehat{\mathbf{B}} \mathbf{p}+\sigma_{n}^{2} \overrightarrow{\mathbf{e}}
$$

where $\mathbf{r}_{0}$ denotes that a virtual ULA with continuous sensors in the range of $[-(M N+M-1), M N+M-1]$. Based on the observation vector of the virtual ULA $\mathbf{r}_{0}$, the covariance matrix of the virtual array can be expressed as

$$
\mathbf{R}_{v}=\mathbf{r}_{0} \mathbf{r}_{0}^{H} .
$$

Because the new vector $\mathbf{r}_{0}$ is a single snapshot signal, the rank of virtual array covariance is one. Thus, estimating the DOA by traditional algorithm is inappropriate. However, the Toeplitz matrix method with the symmetric structure of the steering vector can solve the coherence problem.

We assume that $\left[\mathbf{r}_{0}\right]_{i}\left(-N_{\text {Max }} \leq i \leq N_{\text {Max }}\right)$ denotes $i+N_{\text {Max }}$ row of the vector $\mathbf{r}_{0}$. Obviously, $\left[\mathbf{r}_{0}\right]_{i}=\left[\mathbf{r}_{0}\right]_{-i}^{*}$, and a Toeplitz matrix can be constructed as

$$
\widetilde{\mathbf{R}}_{1}=\left[\begin{array}{cccc}
{\left[\mathbf{r}_{0}\right]_{0}} & {\left[\mathbf{r}_{0}\right]_{-1}} & \cdots & {\left[\mathbf{r}_{0}\right]_{-N_{\mathrm{Max}}}} \\
{\left[\mathbf{r}_{0}\right]_{1}} & {\left[\mathbf{r}_{0}\right]_{0}} & \cdots & {\left[\mathbf{r}_{0}\right]_{-N_{\mathrm{Max}}+1}} \\
\vdots & \vdots & \ddots & \vdots \\
{\left[\mathbf{r}_{0}\right]_{N_{\operatorname{Max}}}} & {\left[\mathbf{r}_{0}\right]_{N_{\mathrm{Max}}-1}} & \cdots & {\left[\mathbf{r}_{0}\right]_{0}}
\end{array}\right] .
$$

In this way, the traditional subspace methods can be employed on the covariance matrix $\mathbf{R}_{1}$ for DOA estimation based on the coprime array.

In order to making full use of redundant virtual array elements and increase the utilization of received signal data, 


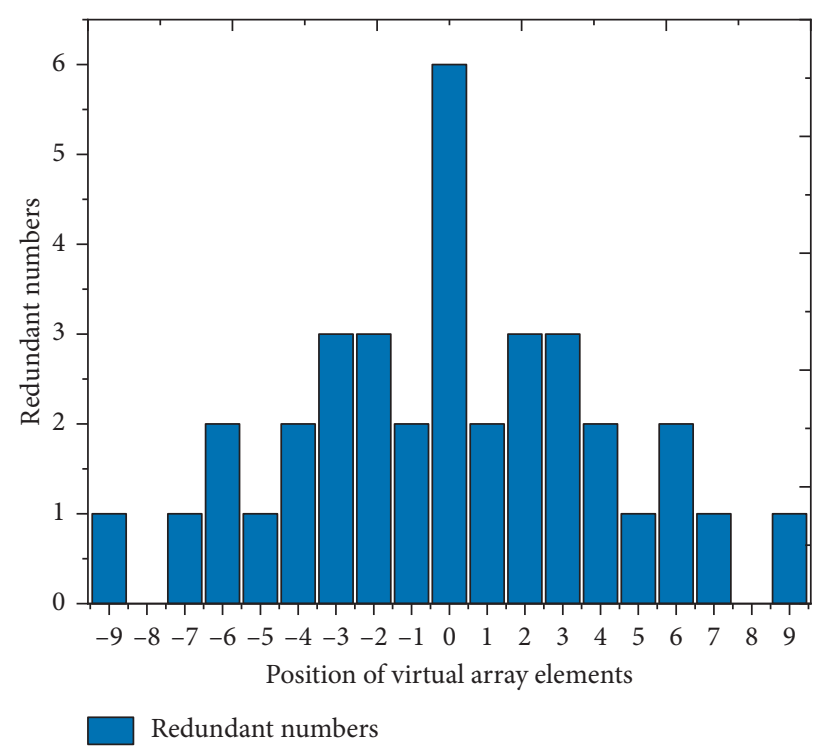

FIGURE 2: Distribution of virtual array.

data of redundant elements are compiled into a new received signal. Section 3.2 will show how the algorithm works.

\subsection{Improved Algorithm Based on Reusing the Redundant} Virtual Array Elements. In order to effectively utilize the received signal information in the redundant virtual array element, we take the average redundant elements in the repeated positions. Moreover, we replace the virtual elements in the original positions with the averaging redundant elements. The process is as follows:

$$
\{-7, \ldots, \underbrace{-3,-3,-3}_{\text {averaging values }}, \underbrace{-2,-2,-2}_{\text {averaging values }} \underbrace{-1,-1}_{\text {averaging values }}, \underbrace{0,0,0,0,0,0}_{\text {averaging values }}, \underbrace{1,1}_{\text {averaging values }}, \underbrace{2,2,2}_{\text {averaging values }}, \underbrace{3,3,3}_{\text {averaging values }}, \ldots, 7\} .
$$

From equation (16), the redundant virtual array elements are fully utilized. Then, we can get a new observation vector $\overline{\mathbf{r}}_{0}$, which is denoted as

$$
\overline{\mathbf{r}}_{0}=\overline{\widehat{\mathbf{B}}} \overline{\mathbf{p}}+\sigma_{n}^{2} \overrightarrow{\mathbf{e}}
$$

In equation (17), the new covariance matrix $\bar{R}$ is reconstructed by restructuring the Toeplitz matrix method. Then, Root-MUSIC algorithm for DOA estimation is introduced with covariance matrix $\bar{R}$. Firstly, we define a polynomial as follows:

$$
f(z)=e_{i}^{H} \mathbf{p}(z), \quad i=K+1, \ldots, N_{\mathrm{Max}},
$$

where $e_{i}$ is $N_{\mathrm{Max}}-K$ smaller eigenvectors of the covariance matrix $\bar{R}$ and $\mathbf{p}(z)=\left[1, z, \ldots, z^{N_{\text {Max }}}\right]^{T}$. Because the signal subspace and noise subspace are orthogonal, equation (18) can be expressed as

$$
f(z)=\mathbf{p}^{H}(z) U_{N} U_{N}^{H} \mathbf{p}(z) .
$$

Considering the conjugate of $z$ existing in $f(z)$, formula (19) can be modified into

$$
f(z)=z^{N_{\mathrm{Max}}-1} \mathbf{p}^{T}\left(z^{-1}\right) U_{N} U_{N}^{H} \mathbf{p}(z) .
$$

Therefore, the DOAs of the targets can be expressed by the following formula:

$$
\theta_{i}=\arcsin \left(\frac{\lambda}{2 \pi d_{0}} \arg \left(z_{i}\right)\right), \quad i=1, \ldots, K
$$

3.3. Algorithm Steps. In order to better illuminate the algorithm ideas, we add detailed algorithm steps in Algorithm 1.

\section{Simulation Results}

In this section, in the coprime array with $M=2$ and $N=3$, we assume that both subarrays start from zero. Obviously, the total physical sensors are $2 M+N-1=6$. We compared the performance of improved algorithm with traditional algorithm based on coprime array and ULA with six $(M=$ 6) physical sensors.

Simulation will show advantages of reusing the redundant virtual array elements in terms of root means square error and spatial spectral resolution. 
Input: received signal $\{\mathbf{X}(t)\}$

Output: $\left\{\widehat{\theta}_{k}\right\}, k=1,2, \ldots, K$

Step 1: after the matched filter, received signal $\{\mathbf{X}(t)\}$ will be obtained.

Step 2: according to the formula $\mathbf{R}=\mathbf{E}\left[\mathbf{X}(t) \mathbf{X}^{H}(t)\right]=\mathbf{A R}_{S} \mathbf{A}^{H}+\sigma^{2} \mathbf{I}_{N \times N}$, the received signal covariance matrix is obtained.

Step 3: the covariance matrix $\mathbf{R}$ is vectorized to obtain the vector matrix $\mathbf{r}$.

Step 4: the virtual array elements in the vector matrix $\mathbf{r}$ are sorted according to the position size.

Step 5: averaging the virtual array elements in the same position.

Step 6: new vector $\bar{r}$ will be obtained.

Step 7: making $\overline{\mathbf{r}}$ become a Toeplitz matrix to overcome the single snapshot effect, then a new covariance matrix $\widetilde{\mathbf{R}}_{1}$ will be obtained.

Step 8: using Root-MUSIC algorithm to estimate DOA of targets.

Algorithm 1: Algorithm steps.

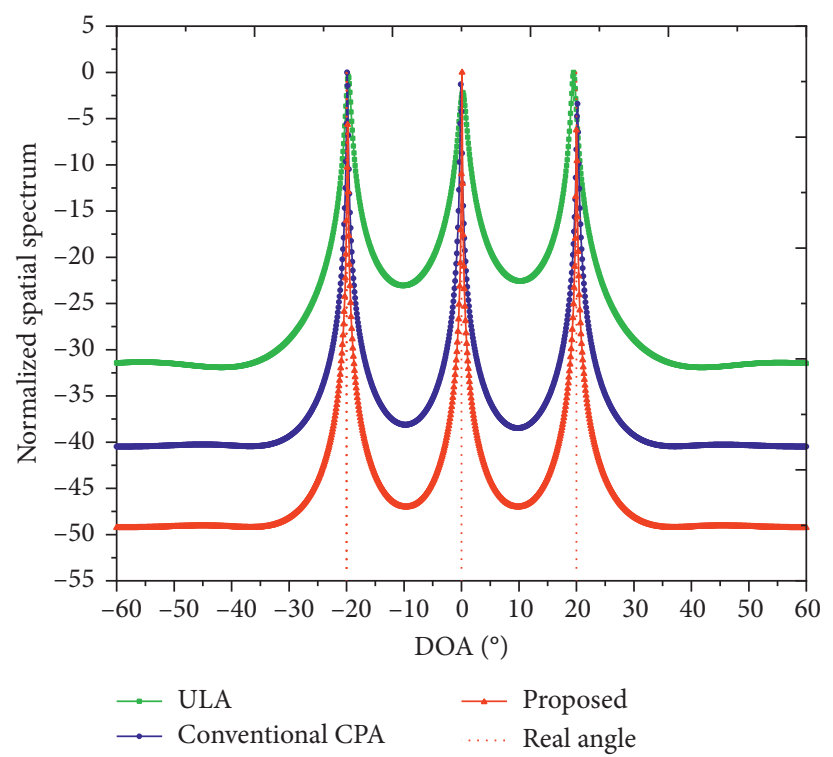

Figure 3: Comparison of resolution performance.

4.1. Simulation of Spatial Spectral Resolution. In the simulation, a pair of coprime integers are assumed as $M=2$ and $N=3$. Similarly, the uniform linear array has $M=6$ physical sensors. Considering three equal power targets impinging on the coprime array from the directions in $\left[-20^{\circ}, 0^{\circ}, 20^{\circ}\right]$ with $\mathrm{SNR}=0 \mathrm{~dB}$, the snapshots is $L=500$. The following formula helps achieve the spatial spectrum:

$$
f_{\text {MUSIC }}(\theta)=\frac{1}{\left\|\mathbf{a}^{H}(\theta) \mathbf{U}_{n}\right\|^{2}},
$$

where $\mathbf{a}(\theta)$ denotes the steering vector of the signal and $\mathbf{U}_{n}$ consists of eigenvectors corresponding to $N_{\text {Max }}-K$ eigenvalues.

Figure 3 shows the resolution performance of the three algorithms. It can be seen that the spectrum peak of the algorithm with redundant virtual array element (red solid line) is sharper, and the side lobe is lower. Thus, the red line represents the best performance. Similarly, the algorithm resolution with conventional CPA structure is better than the ULAs referring to the higher array aperture.
4.2. Relationship between SNR and RMSE. We assume three incoherent signals with equal power impinging on the arrays from the directions $\theta_{1}=10^{\circ}, \theta_{2}=20^{\circ}$, and $\theta_{3}=30^{\circ}$. Then, the SNR varies from $-10 \mathrm{~dB}$ to $10 \mathrm{~dB}$ with an interval of $2 \mathrm{~dB}$. Meanwhile, the number of snapshots is $L=500$, which is the same with that of Monte-Carlo trials. The noise is assumed to be Gaussian white noise with a mean value of 0 and variance of $\sigma^{2}$. In the next all simulations, we will use the Root-MUSIC algorithm to calculate the DOAs of the targets. The formula for calculating root mean square error is defined as

$$
\mathrm{RMSE}=\sqrt{\frac{1}{L K} \sum_{i=1}^{500} \sum_{k=1}^{K}\left[\left(\hat{\theta}_{k}^{i}-\theta_{k}\right)^{2}\right]}
$$

where $K$ denotes the number of the targets, while $L$ denotes the snapshots of the received signal $(i=1,2, \ldots, K)$.

As shown in Figure 4, under the same SNR condition, the root mean square error of the proposed algorithm is the smallest. With the increase of SNR, the algorithm obtains 


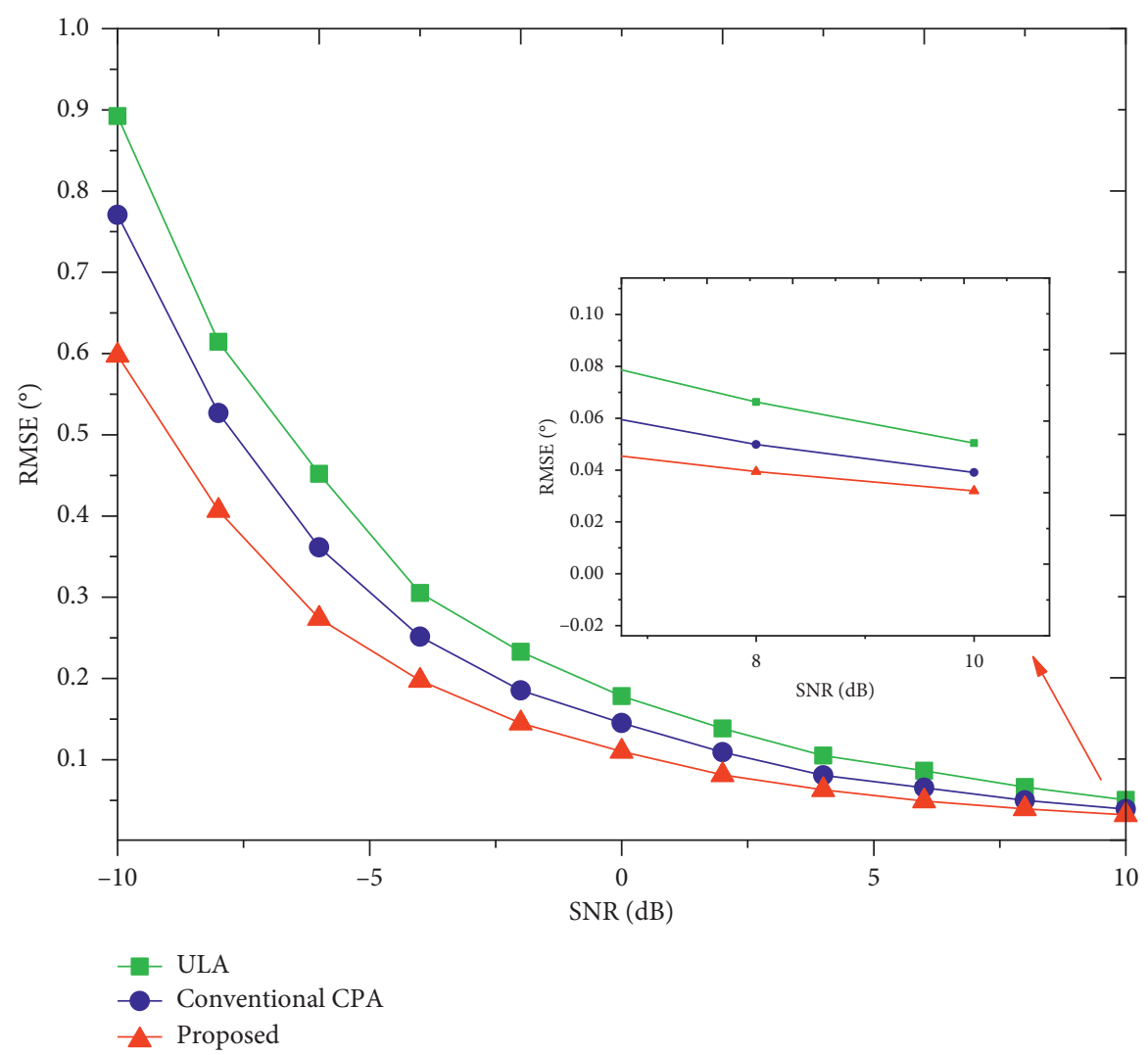

Figure 4: Relationship between SNR and RMSE.

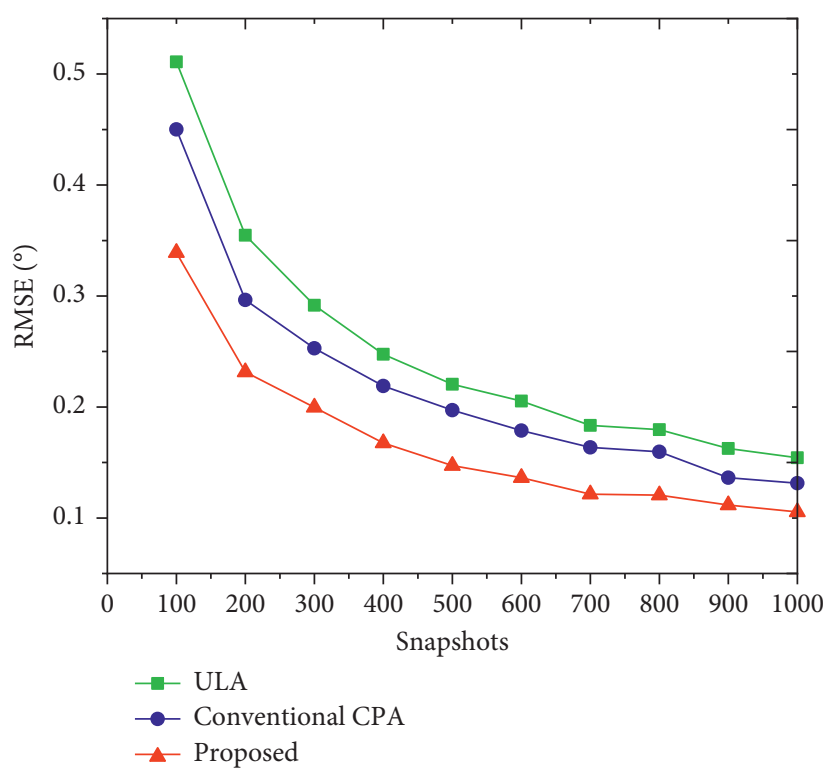

FIGURE 5: Relationship between snapshots and RMSE.

higher estimation accuracy and smaller root mean square error value.

4.3. Relationship between Snapshots and RMSE. Based on the same assumption in part $B$, the $\mathrm{SNR}$ is $0 \mathrm{~dB}$. The number of snapshots $L$ varies from 100 to 1000 with an interval of 100 .
Moreover, the number of Monte-Carlo trials is 500. The noise is assumed to be Gaussian white noise with a mean value of 0 and variance of $\sigma^{2}$.

From Figure 5, with the increase of snapshots, the root means square error plummets. Under the same number of snapshots, the proposed algorithm has better performance. 


\section{Conclusions}

In this paper, a new algorithm via reusing the redundant virtual array elements is proposed for DOA estimation of uncoherent targets. This method averages the virtual array elements in the repeated position. In this way, a new covariance matrix of signal is constructed, which increases the utilization of the received signal data. In addition, RootMUSIC algorithm is employed to estimate the DOAs of these targets. Unlike the existing algorithm, the improved algorithm makes full use of the information in the redundant virtual array elements. Consequently, the proposed algorithm has greatly improved the resolution performance and reduced estimation error. Furthermore, the algorithm can also be introduced to the MARs and NAs based on the conception of difference coarray and sum-difference coarray. Simulation results show that our algorithm provides better performance for uncoherent signals than conventional methods.

\section{Data Availability}

The data supporting the conclusion of the article are included within the article.

\section{Conflicts of Interest}

The authors declare that there are no conflicts of interest regarding the publication of this paper.

\section{Acknowledgments}

This work was supported by the Foundation of National Natural Science Foundation of China under grant numbers 61871395 and 61601504.

\section{References}

[1] A. Moffet, "Minimum-redundancy linear arrays," IEEE Transactions on Antennas and Propagation, vol. 16, no. 2, pp. 172-175, 2003.

[2] P. Pal and P. P. Vaidyanathan, "Nested arrays: a novel approach to array processing with enhanced degrees of freedom," IEEE Transactions on Signal Processing, vol. 58, no. 8, pp. 4167-4181, 2010.

[3] J. Shi, F. Wen, and T. Liu, "Nested MIMO radar: coarrays, tensor modeling and angle estimation," IEEE Transactions on Aerospace and Electronic Systems, 2020.

[4] P. Pal and P. P. Vaidyanathan, "Co-prime sampling and the music algorithm," in Proceedings of the International Conference on Digital Signal Processing, pp. 289-294, Sedona, AZ, USA, January 2011.

[5] C. Zhou, Z. Shi, Y. Gu, and X. Shen, "DECOM: DOA estimation with combined MUSIC for co-prime array," in Proceedings of the International Conference Wireless Communication and Signal Process, pp. 1-5, Hangzhou, China, October 2013.

[6] E. BouDaher, Y. Jia, F. Ahmad, and M. G. Amin, "Multifrequency co-prime arrays for high-resolution direction-ofarrival estimation," IEEE Transactions on Signal Processing, vol. 63, no. 14, pp. 3797-3808, 2015.
[7] S. Qin, Y. D. Zhang, and M. G. Amin, "Generalized co-prime array configurations for direction-of-arrival estimation," IEEE Transactions on Signal Processing, vol. 63, no. 6, pp. 13771390, 2015.

[8] J. Li, D. Jiang, and X. Zhang, "DOA estimation based on combined unitary ESPRIT for coprime MIMO radar," IEEE Communications Letters, vol. 21, no. 1, pp. 96-99, 2017.

[9] C. Zhou, Y. Gu, Y. D. Zhang, Z. Shi, T. Jin, and X. Wu, "Compressive sensing-based coprime array direction-of-arrival estimation," IET Communications, vol. 11, no. 11, pp. 1719-1724, 2017.

[10] C. Zhou, Y. Gu, S. He, and Z. Shi, "A robust and efficient algorithm for coprime array adaptive beamforming," IEEE Transactions on Vehicular Technology, vol. 67, no. 2, pp. 1099-1112, 2018.

[11] E. Boudaher, F. Ahmad, M. G. Amin, and A. Hoorfar, "Mutual coupling effect and compensation in non-uniform arrays for direction-of-arrival estimation," Digital Signal Processing, vol. 61, pp. 3-14, 2017.

[12] J. Liu, Y. Zhang, Y. Lu, S. Ren, and S. Cao, "Augmented nested arrays with enhanced DOF and reduced mutual coupling," IEEE Transactions on Signal Processing, vol. 65, no. 21, pp. 5549-5563, 2017.

[13] C.-L. Liu and P. P. Vaidyanathan, "Remarks on the spatial smoothing step in coarray MUSIC," IEEE Signal Processing Letters, vol. 22, no. 9, pp. 1438-1442, 2015.

[14] Z. Shi, C. Zhou, Y. Gu, N. A. Goodman, and F. Qu, "Source estimation using coprime array: a sparse reconstruction perspective," IEEE Sensors Journal, vol. 17, no. 3, pp. 755-765, 2017.

[15] J. Shi, G. Hu, X. Zhang, F. Sun, and H. Zhou, "Sparsity-based two-dimensional DOA estimation for coprime array: from sum-difference coarray viewpoint," IEEE Transactions on Signal Processing, vol. 65, no. 21, pp. 5591-5604, 2017.

[16] J. Shi, G. Hu, X. Zhang, F. Sun, W. Zheng, and Y. Xiao, "Generalized co-prime MIMO radar for DOA estimation with enhanced degrees of freedom," IEEE Sensors Journal, vol. 18, no. 3, pp. 1203-1212, 2018.

[17] Z. Meng and W. Zhou, "Direction-of-Arrival estimation in Co-prime array using the ESPRIT-based method," Sensors, vol. 19, no. 3, 2019.

[18] Z. Zheng, Y. Huang, W.-Q. Wang, and H. C. So, "Directionof-Arrival estimation of coherent signals via coprime array interpolation," IEEE Signal Processing Letters, vol. 27, no. 99, pp. 585-589, 2020.

[19] C. Wu and C. Ye, "DOA estimation for unfolded Co-prime arrays: successive-MUSIC algorithm," IOP Conference Series Materials Science and Engineering, vol. 719, 2020012035.

[20] P. Gong, X. Zhang, and T. Ahmed, "Computationally efficient DOA estimation for Co-prime linear array: a successive signal subspace fitting algorithm," International Journal of Electronics, vol. 107, no. 8, pp. 1216-1238, 2020. 